\title{
Dimensiones para el estudio de la calidad de servicios en bibliotecas universitarias
}

\author{
Edixson CALDERA MORILLO \\ Centro de Investigación y Desarrollo en Tecnologías del Conocimiento. CIDTEC \\ Universidad del Zulia-Venezuela \\ ejocal@gmail.com \\ Johann PIRELA MORILlO \\ Centro de Investigación y Desarrollo en Tecnologías del Conocimiento. CIDTEC \\ Universidad del Zulia-Venezuela \\ johannpirela@hotmail.com \\ Egla ORTEGA \\ Centro de Investigación y Desarrollo en Tecnologías del Conocimiento. CIDTEC \\ Universidad del Zulia-Venezuela \\ eglaortega@gmail.com
}

Recibido: 14/04/2011

Aceptado: 17/05/2011

\section{RESUMEN}

Se exponen algunas dimensiones que pueden orientar estudios sobre la calidad de servicios en bibliotecas universitarias, asumiendo como premisa los desafíos emergentes para la educación superior, que demandan servicios bibliotecarios, pautados según las necesidades de los usuarios y en sintonía con las demandas de los nuevos modelos curriculares, centrados en el aprendizaje autónomo y permanente de los estudiantes, para lo cual se requiere una mayor integración de los servicios bibliotecarios y de información con los procesos de formación y de investigación de las universidades. La metodología utilizada se apoya en técnicas de investigación documental, combinada con procedimientos de análisis, síntesis, comparación e integración de concepciones y planteamientos. Los resultados señalan que las dimensiones para estudiar la calidad de los servicios son: las expectativas de los usuarios sobre la calidad, la percepción que los usuarios construyen sobre de la calidad de servicio y la satisfacción. Como conclusión se plantea que los estudios sobre la calidad de los servicios en bibliotecas académicas son esenciales en este momento, por cuanto permiten obtener información valiosa sobre las posibles brechas entre lo que los usuarios esperan de los servicios y lo que éstos finalmente reciben, de lo cual se podría deducir la articulación que presentan los servicios con las necesidades de los usuarios y con los desafíos planteados por los modelos curriculares, que están exigiendo mayor protagonismo de los estudiantes en los procesos de formación.

Palabras clave: calidad de servicio, expectativas de los usuarios, percepción de los usuarios, satisfacción de necesidades, bibliotecas académicas. 


\title{
Dimensions for the study of service quality in university libraries
}

\begin{abstract}
Are some dimensions that can guide studies on the quality of services in university libraries, taking as its premise the emerging challenges for higher education, demanding library services, patterned according to the needs of users and in tune with the demands of the new models curriculum, focusing on independent learning and continuing students, for which requires greater integration of library and information services to the processes of teaching and research universities. The methodology is based on documentary research techniques, combined with procedures of analysis, synthesis, comparison and integration of concepts and approaches. The results show that the dimensions to study the quality of services are: user expectations on quality, the perception that users build on the quality of service and satisfaction. In conclusion it is suggested that studies on the quality of services in academic libraries are essential at this time, because it enables valuable information on potential gaps between what users expect from services and what they ultimately receive, of what which could have deduced the joint services with the needs of users and the challenges posed by the curriculum models, which are demanding greater involvement of students in the training process.
\end{abstract}

Key words: service quality, user expectations, user perceptions, needs satisfaction, and academic libraries.

\section{INTRODUCCIÓN}

Este estudio, de tipo teórico y documental, parte de la premisa de que en este momento los modelos curriculares de la educación superior están exigiendo un mayor énfasis en el aprendizaje autónomo del estudiante, para lo cual se requiere que los servicios que se ofrecen en las bibliotecas universitarias deban estar cada vez más alineados con el currículo y con las necesidades y expectativas de los usuarios, quienes, entre otras cosas, deben aprender a agregarle valor a la información para construir conocimiento útil y funcional, que permita el desarrollo de las competencias generales y específicas, para actuar con dinamismo y creatividad en los escenarios globales y complejos de las sociedad del conocimiento. En la Conferencia Mundial sobre Educación Superior, realizada en París (2009), se insistió en la necesidad de cultivar en los estudiantes el pensamiento crítico e independiente y la capacidad de aprendizaje durante toda la vida, lo cual incluye la promoción de la investigación para el desarrollo y el uso de las nuevas tecnologías de información y comunicación con sentido crítico. Igualmente, se expresa la necesidad de que las universidades fortalezcan sus capacidades institucionales para generar programas dirigidos al aprendizaje continuo, estimulando e intensificando el uso de fuentes de bibliotecas y herramientas para sustentar o apoyar la enseñanza, el aprendizaje y la investigación. En el marco de estos argumentos, se proponen tres dimensiones alrededor de las cuales es posible emprender estudios sobre la calidad de los servicios que las bibliotecas universitarias ofrecen a los usuarios, como una forma de expresar la articulación entre la oferta informativa y la demanda. Las dimensiones son: las expectativas de los usuarios sobre la calidad, la percepción que los usuarios construyen sobre de la calidad de servicio y la satisfacción. 
Como conclusión se plantea que los estudios sobre la calidad de los servicios en bibliotecas universitarias son esenciales, por cuanto permiten obtener información valiosa sobre las posibles brechas entre lo que los usuarios esperan de los servicios y lo que éstos finalmente reciben, de lo cual se podría deducir la articulación que presentan los servicios con las necesidades de los usuarios y con los desafíos planteados por los modelos curriculares, que están exigiendo mayor protagonismo de los estudiantes en los procesos de formación.

\section{CONTEXTO DE LA CALIDAD DE LOS SERVICIOS EN BIBLIOTECAS UNIVERSITARIAS. LA NECESIDAD DE SU ESTUDIO}

En la actualidad, los modelos curriculares que están ganando terreno en las universidades están basados en competencias, que le otorgan mayor protagonismo al estudiante y replantean la función del profesor como el único actor que detenta el conocimiento. En contraposición con esta idea, se plantea la necesidad de dotar al estudiante de las herramientas que le permitan asumirse como protagonista de la construcción del conocimiento para transferirlo en la solución de problemas y el aprovechamiento de oportunidades que redunden en mayores grados de desarrollo colectivo. Según la visión de Orera (2005), asistimos a un cambio de enfoque en la enseñanza universitaria, el cual ha pasado de estar centrado en el profesor a otro basado en el aprendizaje del estudiante, lo que requiere el apoyo de variados recursos: informativos, tecnológicos, pedagógicos y otros. Este cambio de enfoque trae consigo el redimensionamiento en la concepción, función y alcance de los servicios bibliotecarios y de información, que deben transitar hacia la incorporación del modelo conocido como Centro de Recursos para el Aprendizaje y la Investigación (CRAI), que supone avanzar hacia una biblioteca integrada de forma activa y participativa en el aprendizaje, la docencia y la investigación universitaria.

La transición de los servicios bibliotecarios tradicionales hacia los centros de recursos para el aprendizaje y la investigación, se ubica, según Area y otros (2007), en el contexto de la transformación de la educación superior, la cual se está desplazando del aprendizaje finalista y "para siempre" al aprendizaje a lo largo de la vida y de la función del profesorado como transmisor de contenidos al facilitador de competencias y mediador de experiencias de aprendizaje, lo cual otorga al estudiante una mayor dedicación y autonomía de trabajo para construir el conocimiento que requiere. La necesidad de una nueva formación, supone que tanto los estudiantes como los docentes cuenten con los espacios y recursos que posibiliten el desarrollo del aprendizaje autónomo, problematizador y crítico. En el contexto de estas tendencias educativas que se plantean para las universidades, surge la necesidad de posicionar a las organizaciones en general y a las organizaciones de servicio como las bibliotecas universitarias, en particular, como centros que deben considerar la percepción que tienen los usuariosclientes sobre los productos-servicios que reciben.

En efecto, para que los clientes den una opinión positiva, la organización debe satisfacer plenamente todas sus necesidades y expectativas. Cuando las organizaciones 
logran este propósito puede hablarse de que sus servicios son de calidad. La calidad, y más concretamente la calidad del servicio, se está convirtiendo en nuestros días en un requisito imprescindible para competir en las organizaciones industriales y comerciales de todo el mundo, ya que las implicaciones que tiene en la cuenta de resultados, tanto en el corto como en el largo plazo, son muy positivas para las empresas envueltas en este tipo de procesos. Considerando la importancia de satisfacer a los clientes, es primordial entonces contar con información adecuada sobre dichas necesidades y expectativas que agrupen aspectos relacionados con los atributos del servicio que determinan el nivel de calidad deseado. Por consiguiente, la calidad del servicio se convierte en un elemento estratégico que otorga una ventaja competitiva a aquellas organizaciones que tratan de alcanzarla. En el mismo orden de idea Pirela y Portillo (2003) afirman que quizás una de las organizaciones más interesadas en la calidad sean las universidades dada la misión de servicio que deben cumplir mediante sus componentes estratégicos. Las universidades, entendidas como organizaciones de conocimiento, deben estructurar y organizar servicios para apoyar sus funciones medulares, las cuales tienen que ver con la docencia, la investigación y la extensión. De modo que son las bibliotecas algunos de los servicios fundamentales de las universidades, ya que es una institución social que posee una estructura organizada y estable de valores, normas y procedimientos que agrupan a personas con el propósito de satisfacer una necesidad social. Según Shera (1990), la biblioteca como institución social tiene la finalidad de servir a la cultura y es un organismo de comunicación, en tanto que el bibliotecólogo desempeña el papel de mediador entre los seres humanos y sus registros gráficos. El papel de la biblioteca no sólo como un componente clave en el sistema social de comunicación sino, fundamentalmente, como parte orgánica de la vida social, económica y cultural de un país y como institución formadora de la conciencia social; es el de servir como base a la educación, al contribuir con la formación del aspecto espiritual y de la conciencia política y social del hombre, y como una institución ideológica y de información científica que orienta la labor educativo-cultural y de información a toda la población. En la biblioteca se realizan actividades que contribuyen a la creación y transmisión de valores o de significados, cuyo fin es modelar el comportamiento social mediante la formación de las personas y su integración a las convenciones y estructuras culturales por medio del libre acceso al conocimiento contenido en los registros gráficos.

La biblioteca es la institución social llamada a orientar a la comunidad hacia la información y a capacitar a ésta para el manejo y aprovechamiento racional de los recursos tecnológicos e informacionales. Desde el punto de vista vital, según el enfoque orteguiano, mejorar las posibilidades del acceso a la información significa, por una parte, capacitar a las personas que integran un grupo social en el desarrollo de habilidades para el manejo de las herramientas necesarias para tener acceso a la información y, por otra, estimularlas para que se haga un uso adecuado, inteligente y creativo, de esta información, como un componente esencial en la toma de decisiones que contribuyen a la formación de la ética personal y colectiva.

En el mismo orden de idea, Lopera (2002) afirma que es diferente la lógica de la empresa o del negocio a la lógica de la biblioteca, aunque ésta sea considerada como 
una organización que debe ser administrada apropiadamente y que presta ciertos servicios -- al igual que las empresas comerciales del sector servicios --, la lógica de la biblioteca no puede ser la misma que la de un negocio, puesto que a éste le interesan fundamentalmente las ganancias monetarias, mientras que la biblioteca como institución social y cultural está atendiendo una necesidad básica que tiene que ver con la formación del intelecto y del espíritu, la cual no entra en la lógica del utilitarismo y del instrumentalismo mercantil, puesto que iría en contravía de la concepción del ser humano como raíz y meta de cualquier tarea. La biblioteca persigue exclusivamente intereses sociales, no económicos como es el caso de los negocios. Sin embargo, entre sus responsabilidades se encuentra, además del cumplimiento básico de las normas económicas y legales, ofrecer un servicio que cubra las expectativas de los usuarios y los motive a seguir empleando esos espacios para enriquecerse académicamente. En este sentido, Arriola (2006) afirma que la calidad del servicio prestado por la biblioteca, va más allá de la organización, conservación y diseminación de información, hay también las que corresponden al intercambio de conocimientos, a la interacción social y cultural con su ámbito de influencia a la formación y educación de quienes producen el conocimiento y ofrecer espacios y servicios adecuados a las necesidades de los usuarios. Igualmente Lopera (2000) afirma que la biblioteca universitaria ha de proponerse, entonces, como un objetivo estratégico de supervivencia, la integración sistemática de las redes de computadoras o telemáticas, de las redes de información y de las redes académicas o científicas, para constituir las redes de conocimiento así como también la excelencia en el servicio.

Para lograr este objetivo la misma debe generar una convergencia organizacional y funcional para la concepción, diseño, implantación, operación y evaluación, y una convergencia social y cultural que permita el desarrollo de un lenguaje común para facilitar el diálogo y las prácticas entre los actores de los diferentes tipos de redes. Dichas convergencias servirán para estudiar la presencia de la calidad de servicio del sistema bibliotecario de las universidades públicas de la región zuliana, estudio éste que podrá contribuir a diseñar estrategias que permitan que los usuarios participen como entes activos de las redes de conocimiento.

\section{SOBRE LA CALIDAD DEL SERVICIO AL CLIENTE}

El servicio al cliente ha tenido gran auge en la gran mayor parte de los países, como un mecanismo de sobrevivencia ante el mercado competitivo, en razón de lo cual se vienen buscando la mejores vías para alcanzar la calidad del servicio que se ofrece al cliente. Por ello, antes de conceptualizar la calidad de servicio, se hace necesario definir el servicio al cliente. Toda empresa debe contar con un conjunto de actividades interrelacionadas que ofrezcan un suministrador con el fin de que el cliente obtenga el producto en el momento y lugar adecuado, para lo cual deberá asegurar un uso correcto del mismo. Partiendo de esta perspectiva, Albrecht y Bradford (1990) plantean que el servicio al cliente comprende todas las actividades que la empresa o sus empleados desarrollan o efectúan para satisfacer a los clientes, tomando decisiones que los 
beneficien. Dentro de este contexto, se debe señalar también, que el servicio al cliente proporciona ingresos, da resultado en muchas formas, como es retener al cliente durante largo tiempo, ya el mismo es una persona con necesidades y preocupaciones que deben estar en primer lugar si un negocio quiere distinguirse por la calidad de servicio. En consecuencia, todas las empresas que han de prestar un servicio como el caso de los sistemas bibliotecarios en las universidades, necesitan contar con una gestión orientada a la satisfacción de sus clientes.

Por ello, para poder mejorar sus servicios, deberán, entre otras cosas, medir su calidad antes de realizar cualquier cambio, por lo que se debe estar bien clarificado y manejarse bien el concepto de medida de la calidad de servicio. Al respecto, Senlle y otros (2001:17) expresan que "la calidad en los servicios es un problema de metodología, trabajo en equipo y relaciones humanas." En cuanto a metodología, plantean estos autores la necesidad de contar con métodos, estrategias y sistemas bien utilizados para recabar información, analizar confiablemente y tomar decisiones para la solución de una determinada problemática existente, en vías de una mejora continua. Aunado a ello, sostienen la importancia de que todos los individuos que conforman la organización deben aprender a cooperar y trabajar unidos, en función no solo de los objetivos institucionales, sino también en adaptarse a cambios continuos y mejoras permanentes. En este orden de ideas, Crosby (1997) define la calidad de servicio como un término que se ha establecido para determinar el nivel de excelencia que la empresa ha tomado para satisfacer a su clientela, siendo aquella que por sus expectativas y necesidades impone a la organización el nivel de calidad que debe alcanzar, mostrando su satisfacción al adquirir el producto.

Se tiene, entonces, que la calidad de servicio al cliente consiste en satisfacer las demandas o exigencias de éste, lo que implica uno de los medios que le permite a la empresa conocer su percepción y expectativa, considerando su funcionamiento en cuanto a la tangibilidad, que implica la adecuación de sus instalaciones, ambiente, equipos, documentos, mobiliario y apariencia de empleado. Asimismo, la confiabilidad del servicio, que implica la prestación de mismo de forma viable, concisa y exacta, lo que tiene inmerso la habilidad del personal, la consistencia de la asistencia, así como la sensación de confianza de lo que se ofrece. De igual forma, la capacidad de respuesta, es decir el estar listo para servir, lo cual tiene implícito, dentro de los sistemas bibliotecarios de las universidades la facilidad de acceso a la información, voluntad y tiempo de servicio, consecución de documentos solicitados, receptividad a la necesidad del cliente, precisión a la necesidad del mismo. Por lo que los empleados de estos servicios, deberán demostrar al cliente seguridad y empatía, es decir, conocimiento del servicio, atención individualizada de las quejas, la cortesía y el respeto a cada usuario.

En función de ello, Senlle y otros (2001) plantean que los objetivos de la calidad de servicio son:

1. Conseguir y mantener la calidad del servicio, para satisfacer de forma permanente las necesidades de los clientes y los requisitos legales.

2. Garantizar a la dirección que la calidad obtenida se mantiene y mejora a lo largo del tiempo y con independencia de las personas que en cada momento formen parte de la plantilla de la empresa. 
3. Tener una base de información con la que se obtengan datos para mejorar continuamente.

4. Aunar y recoger los esfuerzos de todos los miembros de la empresa.

5. Obtener una visión global y particular de los procesos y evaluar su eficacia.

En este sentido, el logro de estos objetivos culminará en la satisfacción al cliente, puesto que es esta actitud la que puede asegurar el futuro de toda empresa u organización. Visto así, la calidad de servicio es un concepto transformacional, pues se puede situar como una filosofía, un proceso de pensamiento o conjunto de valores que tienen implícito una serie de actitudes, métodos, estrategias que requiere el trabajo mancomunado de todos los integrantes de una organización, para orientar sus servicios hacia satisfacer el grado de exigencias del cliente.

\section{ALGUNAS DIMENSIONES PARA ESTUDIAR LA CALIDAD DEL SERVICIO}

\section{Dimensión 1. Expectativas del Cliente}

Las expectativas del cliente, según Zeithaml y Bitner (2002:62) son las "creencias relacionadas con la prestación del servicio que funcionan como estándares o puntos de referencias contra los cuales se juzga su desempeño". Partiendo de esta definición se puede decir que las expectativas del usuario constituyen un elemento esencial a la hora de evaluar la calidad de servicio que prestan los sistemas bibliotecarios de las universidades, por cuanto, se debe conocer lo que requieren los usuarios antes de prestar el debido servicio, para luego conocer la percepción que obtienen del mismo una vez recibido.

En este sentido, los autores antes mencionados plantean que esta es una de las brechas que existen sobre la calidad de servicio, consideran que toda empresa $u$ organización deben orientarse hacia la identificación y medición de ésta dentro de su gestión de servicio, pues ella permite determinar los niveles de satisfacción de los clientes. Para tal efecto ofrecen el siguiente modelo graficado en la figura $\mathrm{N}^{\circ} 1$. 


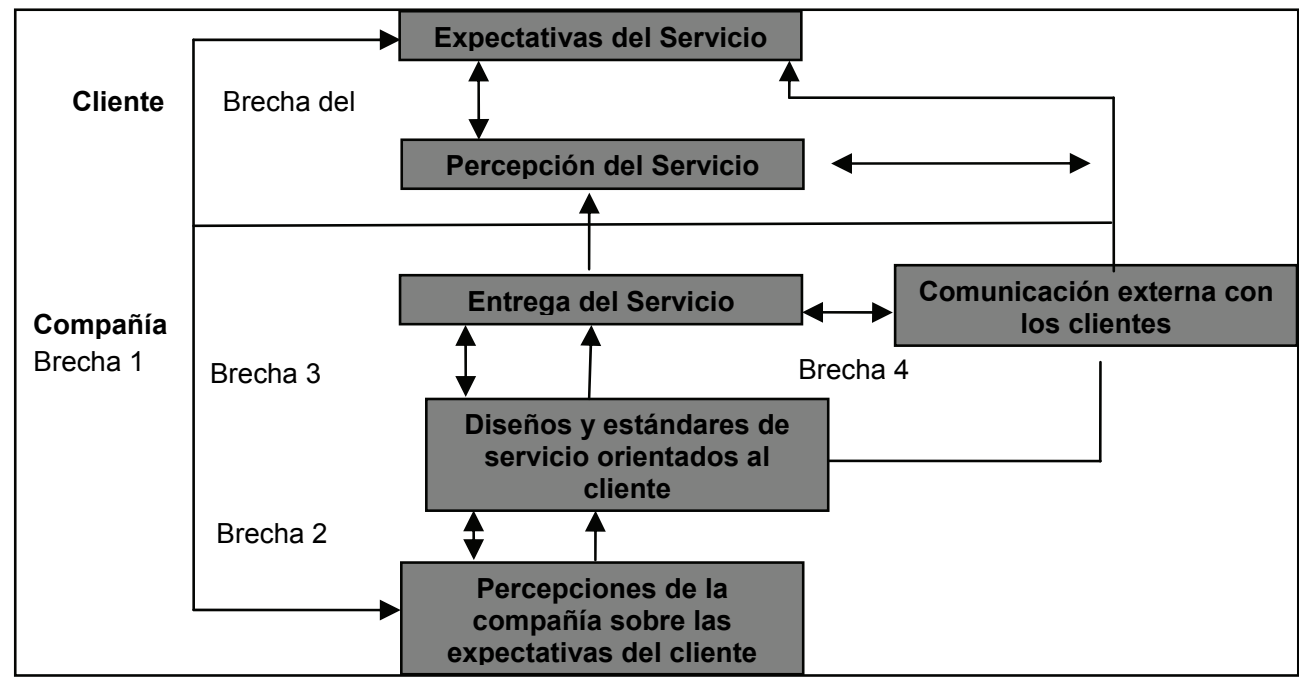

Fuente: Zeithaml, V.; Bitner, M., 2002

FIGURA $\mathbf{N}^{\circ} 1$

MODELO DE LAS BRECHAS SOBRE LA CALIDAD EN EL SERVICIO

Tal y como se observa en la figura, las expectativas del cliente en relación con el servicio están vinculadas con diferentes factores, desde las experiencias que el cliente vive con otras instituciones hasta el efecto de su publicidad sobre el estado psicológico de los clientes en el momento de la prestación del servicio. En términos estrictos, lo que los clientes esperan es tan diverso como su educación, sus valores, experiencias y tipo de expectativa que tienen.

\section{Dimensión 2. Percepción del cliente}

La percepción, desde la perspectiva psicológica, es la capacidad de organizar los datos y la información que llega a través de los sentidos en un todo, creando un concepto. La percepción varía de acuerdo a qué experiencia y a qué aprendizaje previo tenga el observador. Dentro de este contexto, Hoffman y Bateson (2002) definen la percepción del cliente como "la sensación que experimenta un cliente después de recibir un producto o servicio. Esta percepción se forma cuando éste evalúa el paquete de beneficios que le ofrece la empresa, determina la relación costo/beneficio y decide si la sensación es buena". Al tratarse de percepción del cliente, los japoneses la denominan subjetividad social" ya que consiste en la relación entre lo que en verdad es un producto/servicio y lo que representará en un contexto social determinado.

Los aspectos que influyen según Hoffman y Bateson (2002) sobre la persona que percibe son: 
1) Las necesidades y deseos, es decir, la motivación de la persona la cual le hace percibir aquello que le proporcionaría satisfacción.

2) Las expectativas; se tiende a percibir lo que se espera, aquello que resulta más familiar.

3) El estilo de cada persona para enfrentarse al ambiente que lo rodea. Algunas personas perciben más un conjunto de detalles, no pudiendo recordar por separado algunas características específicas del objeto; otras en cambio reparan en tales detalles.

4) La cultura en la que creció, la cual entrena en cierto modo de percibir la realidad.

En síntesis, toda la información y los estímulos que se captan por los sentidos, más aquellos aspectos que influyen en la forma de percibir, generan la elaboración de un concepto sobre el objeto observado como una totalidad. Ahora bien, conociendo estos elementos, se puede observar que cuando un cliente entra a un negocio, su percepción dependerá de una serie de aspectos, tales como la atención que recibe, la calidad del producto, la limpieza del local, la organización del lugar, etc. que luego se agrupará como un todo en el pensamiento del cliente construyendo así una definición de la empresa.

Por esto, es muy importante que los recursos humanos de una empresa, tengan contacto directo con el cliente, tengan claro lo que se quiere transmitir, además de la capacidad para identificar las necesidades del cliente y brindarle apoyo para que logre satisfacerlas.

$\mathrm{Al}$ resolver un problema de servicio o prestación, se debe demostrar al cliente que la empresa se ocupa de él, lo cual será recompensado por éste, siendo leales, por lo tanto, para ocuparse adecuadamente de los clientes, se deben según Zeithaml y Bitner (2002) aplicar las características que se presentan en la figura $\mathrm{N}^{\circ} 2$.

En atención a estos elementos de la percepción del cliente, se tiene que éstos valoran cuándo un determinado usuario le suministran una asistencia o asesoramiento óptimo y acorde a sus requerimientos. Visto así, la calidad del servicio al cliente permite ejecutar una evaluación dirigida que refleja las percepciones del cliente sobre ciertas dimensiones específicas del servicio como: tangibilidad, confiabilidad, capacidad de respuesta, seguridad y empatía. Por su parte, la satisfacción se ve influenciada por las percepciones de la calidad en el servicio, los factores situacionales y personales.

Entonces, si la percepción de la calidad varía de un cliente a otro, y es el mismo quien la determina, ¿cómo se pueden conocer los niveles generales de satisfacción de los clientes para un servicio en particular?. Para Parasuraman, Zeitham y Berry (1998), la clave está en concentrar los esfuerzos de la organización en identificar las necesidades de los clientes y determinar la manera en que estas necesidades afectan la percepción del valor de un servicio. Es por ello, que con el uso de la metodología SERVQUAL se puede comprobar que como cada experiencia y percepción del servicio es particular, entonces se pueden determinar niveles generales de satisfacción mediante la recolección de información acerca de las necesidades de los clientes, la evaluación 
que hacen respecto a diferentes aspectos del servicio brindado y la intención de volver a contratar el mismo servicio.

FIGURA $\mathbf{N}^{\circ} 2$

PERCEPCIÓN DE LA CALIDAD Y SATISFACCIÓN DEL CLIENTE

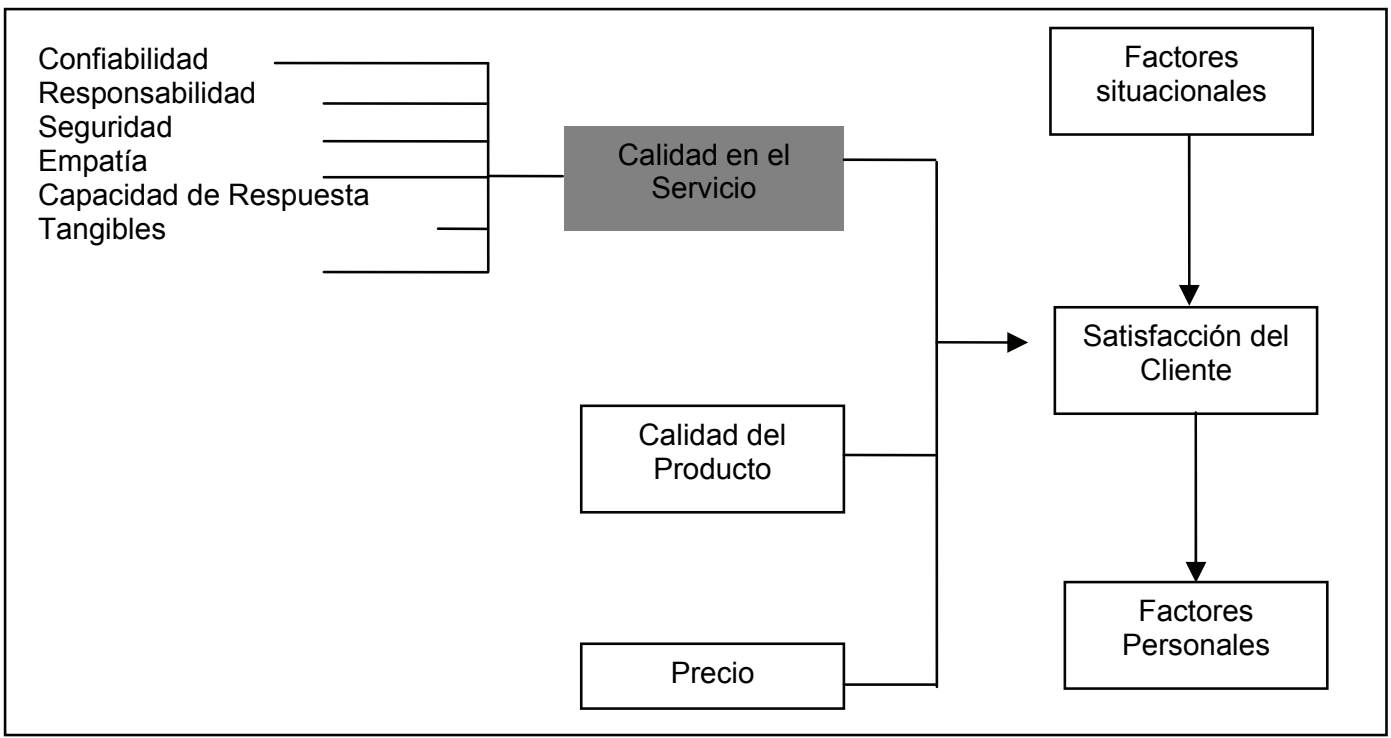

Fuente: Zeithaml, V.; Bitner, M. 2002

En base a la consolidación de esta información se pueden identificar tendencias que indiquen posibles oportunidades de mejora del servicio, así como el impacto que pueden generar en la rentabilidad de la empresa. Esto no es una tarea fácil, por lo que se requieren herramientas especiales que permitan hacer de las mediciones indicadores lo más cercanos posible a la realidad.

La Metodología SERVQUAL, implica que la cuantificación sistemática de la calidad que el cliente percibe de un servicio no es tarea fácil. Se requiere de herramientas que ayuden a las empresas a comprender mejor el significado de valor para el cliente, así como el grado en que sus esfuerzos están cumpliendo con las necesidades y expectativas de los mismos. Esta necesidad llevó al desarrollo de varias técnicas y metodologías para la medición de la satisfacción de los clientes. Una de las más aplicadas en la actualidad, especialmente en empresas norteamericanas, es la metodología SERVQUAL, desarrollada por Zeithaml, Parasuraman y Berry en 1990, a partir de sus estudios realizados para Delivering Quality Service.

Con SERVQUAL, los autores desarrollaron un modelo que identifica las cinco categorías básicas que caracterizan a un servicio, las cuales son representadas en un cuestionario de 22 preguntas. Los datos obtenidos de dicha fuente permiten identificar y 
cuantificar las 5 brechas más importantes que determinan el grado de satisfacción en los clientes, y por lo tanto, la calidad de un servicio.

Para esta metodología, según Parasuraman, Zeitham y Berry (1998), los servicios poseen características especiales, las cuales son tomadas en consideración por los clientes para formarse un juicio respecto a la calidad del mismo. Estas características son integradas en 5 dimensiones generales, las cuales se describen a continuación:

1) Elementos tangibles: representan las características físicas y apariencia del proveedor, es decir, de las instalaciones, equipos, personal y otros elementos con los que el cliente está en contacto al contratar el servicio.

2) Fiabilidad: implica la habilidad que tiene la organización para ejecutar el servicio prometido de forma adecuada y constante.

3) Capacidad de respuesta: representa la disposición de ayudar a los clientes y proveerlos de un servicio rápido.

4) Seguridad (Garantía): son los conocimientos y atención mostrados por los empleados respecto al servicio que están brindando, además de la habilidad de los mismos para inspirar confianza y credibilidad. En ciertos servicios, la seguridad representa el sentimiento de que el cliente está protegido en sus actividades y/o en las transacciones que realiza mediante el servicio.

5) Empatía: es el grado de atención personalizada que ofrecen las empresas a sus clientes.

El nivel de importancia de cada una de estas dimensiones depende tanto del tipo de servicio que ofrece la empresa como del valor que cada una implica para el cliente, lo cual se verá reflejado directamente en los resultados de las encuestas aplicadas a los clientes.

\section{Dimensión 3. Satisfacción del cliente}

En la actualidad, lograr la plena "satisfacción del cliente" es un requisito indispensable para ganarse un lugar en la "mente" de los clientes y por ende, en el mercado meta. Por ello, el objetivo de mantener «satisfecho a cada cliente» ha traspasado las fronteras del departamento de mercadotecnia para constituirse en uno de los principales objetivos de todas las áreas funcionales (producción, finanzas, recursos humanos, etc.) de las empresas exitosas.

Por ese motivo, resulta de vital importancia que tanto mercadólogos, como todas las personas que trabajan en una empresa $\mathrm{u}$ organización, conozcan cuáles son los beneficios de lograr la satisfacción del cliente, cómo definirla, cuáles son los niveles de satisfacción, cómo se forman las expectativas en los clientes y en qué consiste el rendimiento percibido, para que de esa manera, estén mejor capacitadas para coadyuvar activamente con todas las tareas que apuntan a lograr la tan anhelada satisfacción del cliente.

Al respecto, Kotler (2005:40), define la satisfacción del cliente como "el nivel del estado de ánimo de una persona que resulta de comparar el rendimiento percibido de un producto o servicio con sus expectativas". Como se vio en la anterior definición, la satisfacción del cliente está conformada por tres elementos: 
El rendimiento percibido: Se refiere al desempeño (en cuanto a la entrega de valor) que el cliente considera haber obtenido luego de adquirir un producto o servicio. Dicho de otro modo, es el "resultado" que el cliente "percibe" que obtuvo en el producto o servicio que adquirió. El rendimiento percibido tiene las siguientes características:

- Se determina desde el punto de vista del cliente, no de la empresa.

- Se basa en los resultados que el cliente obtiene con el producto o servicio.

- Está basado en las percepciones del cliente, no necesariamente en la realidad.

- Sufre el impacto de las opiniones de otras personas que influyen en el cliente.

Depende del estado de ánimo del cliente y de sus razonamientos. Dada su complejidad, el "rendimiento percibido" puede ser determinado luego de una exhaustiva investigación que comienza y termina en el "cliente".

Las Expectativas: Las expectativas son las "esperanzas" que los clientes tienen por conseguir algo. Las expectativas de los clientes se producen por el efecto de una o más de estas cuatro situaciones:

- Promesas que hace la misma empresa acerca de los beneficios que brinda el producto o servicio.

- Experiencias de compras anteriores.

- Opiniones de amistades, familiares, conocidos y líderes de opinión (p.ej.: artistas).

- Promesas que ofrecen los competidores.

En la parte que depende de la empresa, ésta debe tener cuidado de establecer el nivel correcto de expectativas. Pues para Kotler (2005:41), "si las expectativas son demasiado bajas no se atraerán suficientes clientes; pero si son muy altas, los clientes se sentirán decepcionados luego de la compra".

Un detalle muy interesante sobre este punto es que la disminución en los índices de satisfacción del cliente no siempre significa una disminución en la calidad de los productos o servicios; en muchos casos, es el resultado de un aumento en las expectativas del cliente situación que es atribuible a las actividades de mercadotecnia (en especial, de la publicidad y las ventas personales).

En todo caso, es de vital importancia monitorear "regularmente" las "expectativas" de los clientes para determinar lo siguiente: a) Si están dentro de lo que la empresa puede proporcionarles, b) Si están a la par, por debajo o encima de las expectativas que genera la competencia, c) Si coinciden con lo que el cliente promedio espera, para animarse a comprar.

Los niveles de satisfacción: Luego de realizada la compra o adquisición de un producto o servicio, los clientes experimentan uno de éstos tres niveles de satisfacción:

1) Insatisfacción: Se produce cuando el desempeño percibido del producto no alcanza las expectativas del cliente.

2) Satisfacción: Se produce cuando el desempeño percibido del producto coincide con las expectativas del cliente.

3) Complacencia: Se produce cuando el desempeño percibido excede a las expectativas del cliente.

Dependiendo el nivel de satisfacción del cliente, se puede conocer el grado de lealtad hacia una marca o empresa, por ejemplo: Un cliente insatisfecho cambiará de 
marca o proveedor de forma inmediata (deslealtad condicionada por la misma empresa). Por su parte, el cliente satisfecho se mantendrá leal; pero, tan solo hasta que encuentre otro proveedor que tenga una oferta mejor (lealtad condicional). En cambio, el cliente complacido será leal a una marca o proveedor porque siente una afinidad emocional que supera ampliamente a una simple preferencia racional (lealtad incondicional).

Por ese motivo, según Kotler y Armstrong (2005:11) "las empresas inteligentes buscan complacer a sus clientes mediante prometer solo lo que pueden entregar, y entregar después más de lo que prometieron". Por ello, el motivar a los empleados se traduce en resultados positivos para la empresa, pues el trabajador realiza sus actividades con mayor eficiencia y calidad; además de adquirirle un compromiso más firma.

Las formas teóricas de la motivación sirven para que la organización implemente mejores incentivos y logre así un desempeño en el trabajo más eficaz. Entre estas teorías están las de necesidades prioritariamente estudiadas por Maslow quien se abocó a elaborar un método donde el hombre pudiera desarrollar todas sus capacidades. Este método permitió analizar la motivación como impulsos separados y diferentes.

Una manera de tener éxito es tomar en cuenta las técnicas y herramientas para satisfacer el cliente, las cuales son:

- El trato con personas difíciles. En cualquier organización tarde o temprano se encuentran personas difíciles, la mejor forma de tratar con ellas, es acercarse a las mismas profesionalmente, manteniendo la atención sobre los asuntos que se discuten.

- Identificar la extenuación. La extenuación es una gran amenaza a la satisfacción del cliente, puesto que si existe agotamiento, será difícil pensar razonablemente en el trabajo.

- Obtener más cooperación. Con la cooperación hay una gran diferencia en el trabajo, El secreto es lograr tratar a cada persona en su organización como si fuera el más importante de sus clientes.

- Dirigirse a dar satisfacción al cliente, no sólo un servicio.

Una organización al considerar estas técnicas y herramientas, lograra tener satisfecho a sus clientes, garantizándole la permanencia en el mercado.

\section{CONCLUSIÓN}

Los servicios bibliotecarios de las universidades deben responder con dinamismo al reto que les impone los nuevos modelos curriculares, centrados en la participación del estudiante en los procesos de desarrollo de competencias para actuar proactivamente en los escenarios globales de la sociedad del conocimiento. Las bibliotecas universitarias deben dar el salto cualitativo para convertirse en centros de recursos para el aprendizaje y la investigación, lo cual requiere a su vez que la oferta de servicios esté pautada según las necesidades y expectativas de los usuarios-clientes. 
Por ello, se hace imperiosa la necesidad de realizar estudios sobre la calidad de los servicios que este tipo de biblioteca ofrece, con objeto de saber hasta qué punto están cubriendo las necesidades de aprendizaje e investigación de los usuarios. Algunas de las dimensiones que pueden orientar los estudios sobre la calidad de los servicios de las bibliotecas universitarias son: las expectativas de los usuarios sobre la calidad, la percepción que los usuarios construyen sobre de la calidad de servicio y la satisfacción.

Los estudios sobre la calidad de los servicios en bibliotecas universitarias son esenciales en este momento, por cuanto permiten obtener información valiosa sobre las posibles brechas entre lo que los usuarios esperan de los servicios y lo que éstos finalmente reciben, de lo cual se podría deducir la articulación que presentan los servicios con las necesidades de los usuarios y con los desafíos planteados por los modelos curriculares, que están exigiendo mayor protagonismo de los estudiantes en los procesos de formación.

\section{BIBLIOGRAFÍA}

AREA, M. y otros (2007). De la biblioteca al centro de recursos para el aprendizaje y la investigación. Barcelona-España :Octaedro.

ALBRECHT, Karl y Bradford, J. L. (1990). La excelencia en el servicio: como identificar y satisfacer las expectativas y necesidades del cliente. Bogotá : Legis. $236 \mathrm{p}$.

ARRIOLA NAVARRETE, Óscar. (2006). Evaluación de bibliotecas: un modelo desde la óptica de los sistemas de gestión de calidad. Buenos Aires : Alfagrama. 128 p.

CROSBY, Phillip. (1997). La calidad no cuesta. México : CECSA.

HOFFMAN, K. Douglas y BATESON, John E. G. (2002). Fundamentos de marketing de servicios : conceptos, estrategias y casos. México : Thomson. 569 p.

KOTLER, Philip (2005) Dirección de mercadotecnia. 8 a. ed. México: Prentice Hall. p. 40-41.

KOTLER, Philip y ARMSTRONG, Gary (2005) Fundamentos de marketing. 6 a ed. México : Prentice Hall. p. 10- 11.

LOPERA LOPERA, Luis Hernando. (2002). "Una ética bibliotecológica para afrontar los retos de nuestro tiempo". Revista Interamericana de Bibliotecología. 25(1):6588.

ORERA, L. (2005). La Biblioteca Universitaria: concepto, funciones y retos futuros. En: Orera Orera, Luisa (Edit). La Biblioteca Universitaria. Análisis en su entorno híbrido. Madrid-España. Editorial Síntesis.

PARASURAMAN, A., Zeithaml, V. y Berry, L. (1985). "A Conceptual Model of Service Quality and its Implications for Future Research". Journal of Marketin., 49:41-50

PÍRELA, Johan y PORTILLO, Lisbeth. (2003). "Metodología de evaluación institucional participativa: una estrategia para la transformación universitaria : caso Escuela de Bibliotecología y Archivología de la Universidad del Zulia". Agenda Académica. 9(2):45-60. 
SENLLE, Andrés, MARTÍNEZ, Eduardo y MARTÍNEZ, Nicolás. (2001). Iso 9000.2000 calidad en los servicios. Barcelona, España: Gestión 2000. 186 p.

SHERA, Jesse. (1990). Los fundamentos de la educación bibliotecológica. México: UNAM, CUIB. 520 p.

UNESCO (2009). Conferencia mundial sobre Educación Superior. Las nuevas dinámicas de la educación superior y la investigación para el cambio social y el desarrollo. Disponible en línea, traducido del inglés por el Prof. Miguel Ángel Aquino Benitez. Disponible en línea: http://www.unp.edu.py/priexu/DFCMES.pdf. Consulta: 30-11-09.

ZEITHAML, Valerie A. y Bitner, Mary Jo. (2002). Marketing de servicios: un enfoque de integración del cliente a la empresa. México: McGraw-Hill. 747 p.

ZEITHAML, Valerie A., Parasuraman, A. y Berry, Leonard L. (1993). Calidad total en la gestión de los servicios: como lograr el equilibrio entre las percepciones y las expectativas de los consumidores. Madrid: Díaz de Santos. 